\title{
Method of Averaging for Some Parabolic Partial Differential Equations
}

\author{
Mahmoud M. El-Borai (Corresponding Author) \\ Department of Mathematics and Computer Science, Faculty of Science, Alexandria University, Alexandria, Egypt \\ Email: m_m_elborai@yahoo.com
}

\section{Hamed Kamal Awad}

Department of Mathematics, Faculty of Science, Damanhour University, Damanhour, Egypt

\section{Randa Hamdy M. Ali}

Department of Mathematics, Faculty of Science, Damanhour University, Damanhour, Egypt
Article History

Received: December 27, 2019

Revised: January 23, 2020

Accepted: January 28, 2020

Published: January 31, 2020

Copyright (C) 2020 ARPG \&

Author

This work is licensed under

the Creative Commons

Attribution International (c) $(6)$

Commons Attribution

License 4.0

\begin{abstract}
Quantitative and qualitative analysis of the Averaging methods for the parabolic partial differential equation appears as an exciting field of the investigation. In this paper, we generalize some known results due to Krol on the averaging methods and use them to solve the parabolic partial differential equation.
\end{abstract}

Keywords: Averaging; Averaging method; Partial differential equation; Parabolic partial differential equation.

\section{Introduction}

The investigation in the field of the qualitative and quantitative analysis of the Averaging methods for the parabolic partial differential equation is more exciting field to be studied. We study the parabolic partial differential equation in this paper using the technique of the averaging method of the linear operator. In section 2, we study the averaging of the linear operator where we generalize some known results due to Krol [1]. We consider the following parabolic partial differential equation in the form:

$$
\begin{aligned}
& \frac{\partial u(x, t)}{\partial t}=\varepsilon L(x, t, D) u(x, t), \\
& u(x, 0)=\varphi(x),
\end{aligned}
$$

where

$$
L(x, t, D)=\sum_{|q| \leq 2 m} a_{q}(x, t) D^{q},
$$

$\varepsilon>0, x \in \mathcal{R}^{n}, \mathcal{R}^{n}$ is the $n$-dimensional Euclidean space, $q=\left(q_{1} \ldots q_{n}\right)$ is an $n$-dimensional multi index, $|q|=q_{1}+\cdots+q_{n}, D^{q}=D^{q_{1}} \ldots D^{q_{n}}, D_{j}=\frac{\partial}{\partial x_{j}}$. The coefficients $\mathrm{a}_{\mathrm{q}}(\mathrm{x}, \mathrm{t})$ and $\varphi(\mathrm{x})$ are bounded continuous with bounded derivatives and $\mathrm{D}^{\mathrm{q}} \mathrm{u}(\mathrm{x}, \mathrm{t})$ are bounded on $\mathrm{x} \in \mathcal{R}^{\mathrm{n}}, 0 \leq \mathrm{t} \leq \mathrm{T}$. In section 3 , we discuss a special case for the problem (1), (2). Compare [2-11].

\section{Averaging a Linear Operator}

By averaging the coefficients $a_{\mathrm{q}}(x, t)$ over $t$, we can average the operator $L$

$$
\bar{a}_{q}(x)=\frac{1}{T} \int_{0}^{T} a_{q}(x, t) D^{q} d t,
$$

for all $(x, t), x \in \mathcal{R}^{n}$ producing the averaged operator $\bar{L}(x, D)$ and all the coefficients $\bar{a}_{q}(x),|q| \leq 2 m$ are bounded continuous with bounded derivatives on $\mathcal{R}^{n}$.

$$
\bar{L}(x, D)=\frac{1}{T} \int_{0}^{T} \sum_{|q| \leq 2 m} a_{q}(x, t) D^{q} d t,
$$

like as an approximating problem for (1), (2), we take

$$
\begin{aligned}
& \frac{\partial u^{*}(x, t)}{\partial t}=\varepsilon \bar{L}(x, D) u^{*}(x, t), \\
& u(x, 0)=\varphi(x),
\end{aligned}
$$

another straightforward analysis display the existence and uniqueness of the solutions of problems (1), (2) and (5), (6) on the time-scale $\frac{1}{\varepsilon}$. by

We consider the domain $A=\mathcal{R}^{n} \times[0, T]$. The norm $\|.\|_{\infty}$ is defined by the supremum norm on $A$ and denoted 


$$
\|u(x, t)\|_{\infty}=\sup _{A}|u(x, t)| .
$$

\subsection{Theorem}

Let $\mathrm{u}(\mathrm{x}, \mathrm{t})$ be the solution of the initial value problem $(1),(2)$ and $\mathrm{u}^{*}(\mathrm{x}, \mathrm{t})$ be the solution of the initial value problem (5), (6), then we have the estimate $\left\|u(x, t)-u^{*}(x, t)\right\|_{\infty}=O(\varepsilon)$ on the time-scale $\frac{1}{\varepsilon}$.

Proof. We consider the near-identity transformation:

$$
\widehat{u}(x, t)=u^{*}(x, t)+\varepsilon \int_{0}^{t}(L(x, s, D)-\bar{L}(x, D)) d s u^{*}(x, t),
$$

we have

$$
\left\|\hat{u}(x, t)-u^{*}(x, t)\right\|_{\infty}=O(\varepsilon) \text { on the time-scale } \frac{1}{\varepsilon} .
$$

Differentiation of the near-identity transformation (7) and using the equations (5), (7), we get

$$
\begin{gathered}
\frac{\partial \hat{u}(x, t)}{\partial t}=\frac{\partial u^{*}(x, t)}{\partial t}+\varepsilon(L(x, t, D)-\bar{L}(x, D)) u^{*}(x, t)+\varepsilon \int_{0}^{t}(L(x, s, D)-\bar{L}(x, D)) d s \frac{\partial u^{*}(x, t)}{\partial t} \\
=\varepsilon L(x, t, D) \hat{u}(x, t)-\varepsilon^{2} L(x, t, D) \int_{0}^{t}(L(x, s, D)-\bar{L}(x, D)) d s u^{*}(x, t) \\
+\varepsilon^{2} \bar{L}(x, D) \int_{0}^{t}(L(x, s, D)-\bar{L}(x, D)) d s u^{*}(x, t) \\
=\varepsilon L(x, t, D) \hat{u}(x, t) \\
+\varepsilon^{2} \int_{0}^{t}[(L(x, s, D)-\bar{L}(x, D)) \bar{L}(x, D)-L(x, t, D)(L(x, s, D)-\bar{L}(x, D))] d s u^{*}(x, t) \\
=\varepsilon L(x, t, D) \hat{u}(x, t)+\varepsilon^{2} \mathcal{M}(x, t, D) u^{*}(x, t),
\end{gathered}
$$

with initial value $\hat{u}(x, 0)=\varphi(x)$, where

Let

$$
\begin{aligned}
\mathcal{M}(x, t, D)= & \int_{0}^{t}[(L(x, s, D)-\bar{L}(x, D)) \bar{L}(x, D) \\
& -L(x, t, D)(L(x, s, D)-\bar{L}(x, D))] d s .
\end{aligned}
$$

we obtain

$$
\frac{\partial}{\partial t}-\varepsilon L(x, t, D)=\mathcal{L}
$$

$\mathcal{L} u^{*}(x, t)=O(\varepsilon)$ on the time-scale $\frac{1}{\varepsilon}$,

then

$\mathcal{L}\left(\hat{u}(x, t)-u^{*}(x, t)\right)=O(\varepsilon)$ on the time-scale $\frac{1}{\varepsilon}$.

Moreover $\hat{u}(x, 0)-u^{*}(x, 0)=0$. To end the proof we use barrier functions see [12].

Let $c=\left\|\mathcal{M}(x, t, D) u^{*}(x, t)\right\|_{\infty}$, we introduce the barrier function

and the functions (we omit the arguments)

$$
B(x, t)=\varepsilon^{2} c t,
$$

We get

$$
Z_{1}(x, t)=\hat{u}(x, t)-u(x, t)-B(x, t), Z_{2}(x, t)=\hat{u}(x, t)-u(x, t)+B(x, t) .
$$

$\leq 0$,

$$
\begin{aligned}
& \mathcal{L} Z_{1}(x, t)=\left(\frac{\partial}{\partial t}-\varepsilon L(x, t, D)\right)[\hat{u}(x, t)-u(x, t)-B(x, t)] \\
& =\varepsilon^{2} \mathcal{M}(x, t, D) u^{*}(x, t)-\varepsilon^{2}\left\|\mathcal{M}(x, t, D) u^{*}(x, t)\right\|_{\infty}+\varepsilon^{3} c t L(x, t, D)
\end{aligned}
$$

$Z_{1}(x, 0)=0$ and similarly $\mathcal{L} Z_{2}(x, t) \geq 0, Z_{2}(x, 0)=0$.

$Z_{1}(x, t)$ and $Z_{2}(x, t)$ are bounded, resulting in $Z_{1}(x, t) \leq 0$ and $Z_{2}(x, t) \geq 0$. It follows that

$$
\begin{gathered}
-B(x, t) \leq \hat{u}(x, t)-u(x, t) \leq B(x, t), \\
-\varepsilon^{2} c t \leq \hat{u}(x, t)-u(x, t) \leq \varepsilon^{2} c t,
\end{gathered}
$$

so we can estimate

$$
\|\hat{u}(x, t)-\mathrm{u}(x, t)\|_{\infty} \leq\|B(x, t)\|_{\infty}=O(\varepsilon),
$$

on the time-scale $\frac{1}{\varepsilon}$. We can use the triangle inequality to have

$$
\left\|u(x, t)-u^{*}(x, t)\right\|_{\infty} \leq\left\|\hat{u}(x, t)-u^{*}(x, t)\right\|_{\infty}+\|\hat{u}(x, t)-\mathrm{u}(x, t)\|_{\infty}
$$

$=O(\varepsilon)$ on the time-scale $\frac{1}{\varepsilon}$

\section{A Special Case}

Consider the partial differential equation: 
where

$$
\begin{aligned}
& \frac{\partial u(x, t)}{\partial t}=\varepsilon L_{1}(D) u(x, t), \\
& u(x, 0)=\varphi(x)
\end{aligned}
$$

$$
L_{1}(D)=\left(D_{1}^{2}+\cdots+D_{n}^{2}\right)^{2 N+1}
$$

$N$ is a sufficiently large positive integer.

Let $C_{b}\left(\mathcal{R}^{n}\right)$ is the set of all bounded continuous functions on $\mathcal{R}^{n}$.

Consider the following Cauchy problem [6]:

$$
\begin{aligned}
& \frac{\partial u(x, t)}{\partial t}=\left(D_{1}^{2}+\cdots+D_{n}^{2}\right)^{2 N+1} u(x, t), \\
& u(x, 0)=\varphi(x) \in C_{b}\left(\mathcal{R}^{n}\right) .
\end{aligned}
$$

The solution of the Cauchy problem (11), (12) is given by:

$$
u(x, t)=\int_{\mathcal{R}^{n}} G(x-y, t) \varphi(y) d y,
$$

where the function $G$ is the fundamental solution of the Cauchy problem (11), (12) and $d y=d y_{1} \ldots d y_{n}$. For sufficiently large $N$, we find $\gamma \in(0,1)$ and a constant $M>0$ such that:

$$
\max _{x}\left|D^{q} u(x, t)\right| \leq \frac{M}{t^{\gamma}} \max _{x}|\varphi(x)|
$$

for all $t>0,|q| \leq m$. Like as an approximating problems for (8), (9), we take

$$
\begin{aligned}
& \frac{\partial u^{*}(x, t)}{\partial t}=\varepsilon \bar{L}_{1}(D) u^{*}(x, t), \\
& u^{*}(x, 0)=\varphi(x),
\end{aligned}
$$

where

$$
\bar{L}_{1}(D)=L_{1}(D)
$$

another straightforward analysis displays the existence and uniqueness of the solutions of problems (8), (9) and (13), (14) on the time-scale $\frac{1}{\varepsilon}$.

\subsection{Theorem}

Let $u(x, t)$ be the solution of the initial value problem $(8),(9)$ and $u^{*}(x, t)$ be the solution of the initial value problem (13), (14), then we have the estimate $\left\|u(x, t)-u^{*}(x, t)\right\|_{\infty}=0$.

Proof. By using the near-identity transformation (7), we have

$$
\left\|\hat{u}(x, t)-u^{*}(x, t)\right\|_{\infty}=0 .
$$

Differentiation of the near-identity transformation (7) and using the equations (7), (13), we have

with initial value $\hat{u}(x, 0)=\varphi(x)$,

$$
\frac{\partial \hat{u}(x, t)}{\partial t}=\frac{\partial u^{*}(x, t)}{\partial t}=\varepsilon L(x, t, D) \hat{u}(x, t),
$$

Let

we get

$$
\frac{\partial}{\partial t}-\varepsilon L_{1}(D)=\mathcal{L}_{1}
$$

$\mathcal{L}_{1}\left(\hat{u}(x, t)-u^{*}(x, t)\right)=0$.

$$
\mathcal{L}_{1} \hat{u}(x, t)=0,
$$

Moreover $\hat{u}(x, 0)-u^{*}(x, 0)=0$. We introduce the barrier function

and the functions (we omit the arguments)

$$
B_{1}(x, t)=0 \text {, }
$$

$Z_{3}(x, t)=\hat{u}(x, t)-u(x, t)-B_{1}(x, t), Z_{4}(x, t)=\hat{u}(x, t)-u(x, t)+B_{1}(x, t)$.

We get

$$
\begin{gathered}
\mathcal{L}_{1} Z_{3}(x, t)=\left(\frac{\partial}{\partial t}-\varepsilon L_{1}(D)\right)\left[\hat{u}(x, t)-u(x, t)-B_{1}(x, t)\right] \\
=0
\end{gathered}
$$

$Z_{3}(x, 0)=0$ and similarly $\mathcal{L}_{1} Z_{4}(x, t)=0, Z_{4}(x, 0)=0$.

$Z_{3}(x, t)$ and $Z_{4}(x, t)$ are bounded, resulting in $Z_{3}(x, t)=0$ and $Z_{4}(x, t)=0$. It follows that

so

$$
\hat{u}(x, t)-u(x, t)=0 \text {, }
$$

$\|\hat{u}(x, t)-\mathrm{u}(x, t)\|_{\infty}=0$.

We can use the triangle inequality to obtain

$$
\left\|u(x, t)-u^{*}(x, t)\right\|_{\infty}=0 .
$$

\section{Conclusion}

This paper is focused on generalizing some known results due to Krol on the averaging methods to solve the parabolic partial differential equation. As a special case Cauchy problem is solved for the parabolic partial differential equation. 


\section{Acknowledgment}

The Authors would like to express their sincere gratitude to the chaif editor and the anonymous refrees for their valuable comments and suggestions.

\section{References}

[1] Krol, M. S., 1991. "On the averaging method in nearly time-periodic advection-diffusion problems." SIAM J. Appl. Math., vol. 51, pp. 622-1637.

[2] Ben, L. A. and Ellison, J. A., 1985. "Method of averaging and the quantum anharmonic oscillator." Phys. Rev. Lett., vol. 55, pp. 1950-1953.

[3] Friedman, A., 1964. Partial differential equations of parabolic type. Prentice-Hall, Englewood Cliffs, NJ.

[4] Mahmoud, M. E.-B., Hamed, K. A., and Randa, H. M. A., 2019. "A parabolic transform and averaging methods for general partial differential equations." Journal of Advances in Mathematics, vol. 17, pp. 352361.

[5] Mahmoud, M. E.-B. and Khairia, E.-S. E.-N., 2015. "A parabolic transform and some stochastic Ill-posed problem." British Journal of Mathematics and Computer Science, vol. 9, pp. 418-426.

[6] Mahmoud, M. E.-B. and Khairia, E.-S. E.-N., 2019. "On the solutions of Ill-posed Cauchy problems for some singular integro-partial differential equations." Global Journal of Mathematics, vol. 13, pp. 899-905.

[7] Mahmoud, M. E.-B., Khairia, E.-S. E.-N., and Eman, G. E.-A., 2010. "On some fractional evolution equations." Computers and Mathematics with Applications, vol. 59, pp. 1352-1355.

[8] Mahmoud, M. E.-B., Khairia, E.-S. E.-N., and Hoda, A. F., 2010. "On some fractional stochastic delay differential equations." Computers and Mathematics with Applications, vol. 59, pp. 1165-1170.

[9] Mahmoud, M. E.-B., Moustafa, O. L., and Michael, F. H., 1999. "On the correct formulation of a nonlinear differential equations in Banach space." Int. J. Math., vol. 22,

[10] Mahmoud, M. E.-B., Wagdy, G. E.-S., and Faez, N. G., 2016. "On the Cauchy problem for some parabolic fractional partial differential equations with time delays." Journal of Mathematics and System Science, vol. 6, pp. 194-199.

[11] Verhulst, F., 1999. "On averaging methods for partial differential equations." In SPT98-Symmetry and Perturbation Theory II, A.Degasperis and G.Gaeta eds., World Scientifc. pp. 79-95.

[12] Protter, M. H. and Weinberger, H. F., 1967. Maximum principles in differential equations. Prentice-Hall, Englewood Cliffs, NJ. 